\title{
Less Lumens-Less Risk: A Pilot Intervention to Increase the Use of Single-Lumen Peripherally Inserted Central Catheters
}

David Bozaan, MD 1,2; Deanna Skicki, BSN, RN, CRNI, VA-BC 3 ; Adamo Brancaccio, PharmD4; Ashley Snyder, $\mathrm{MPH}^{1}$; Sue Friebe, MSN, RN, CRNI'; Matthew Tupps, PharmD, MHA ${ }^{4}$; David Paje, MD, MPH'1,2; Vineet Chopra, MD, MSc ${ }^{1,2 \star}$

${ }^{1}$ Division of Hospital Medicine, Department of Internal Medicine, Michigan Medicine, Ann Arbor, Michigan; ${ }^{2}$ A A Ann Arbor Health System, Ann Arbor, Michigan; ${ }^{3}$ Vascular Access Service Team, Michigan Medicine, Ann Arbor, Michigan; ${ }^{4}$ Department of Pharmacy, Michigan Medicine, Ann Arbor, Michigan.

To reduce risk of complications, existing guidelines recommend use of peripherally inserted central catheters (PICCs) with the minimal number of lumens. This recommendation, however, is difficult to implement in practice. We conducted a pilot study to increase the use of single-lumen PICCs in hospitalized patients. The intervention included (1) education for physicians, pharmacists, and nurses; (2) changes to the electronic PICC order-set that set single lumen PICCs as default; and (3) criteria defining when use of multilumen PICCs is appropriate. The intervention was supported by realtime monitoring and feedback. Among 226 consecutive
PICCs, $64.7 \%$ of preintervention devices were single lumen versus $93.6 \%$ postintervention $(P<.001)$. The proportion of PICCs with an inappropriate number of lumens decreased from $25.6 \%$ preintervention to $2.2 \%$ postintervention $(P<.001)$. No cases suggesting inadequate venous access or orders for the placement of a second PICC were observed. Implementing a single-lumen PICC default and providing education and indications for multilumen devices improved PICC appropriateness. Journal of Hospital Medicine 2019;14:42-46. Published online first October 31, 2018. (C) 2019 Society of Hospital Medicine
V ascular access is a cornerstone of safe and effective medical care. The use of peripherally inserted central catheters (PICCs) to meet vascular access needs has recently increased. ${ }^{1,2}$ PICCs offer several advantages over other central venous catheters. These advantages include increased reliability over intermediate to long-term use and reductions in complication rates during insertion., ${ }^{3,4}$

Multiple studies have suggested a strong association between the number of PICC lumens and risk of complications, such as central-line associated bloodstream infection (CLABSI), venous thrombosis, and catheter occlusion. . $^{5-8,9,10-12}$ These complications may lead to device failure, interrupt therapy, prolonged length of stay, and increased healthcare costs. ${ }^{13-15}$ Thus, available guidelines recommend using PICCs with the least clinically necessary number of lumens. ${ }^{1,16}$ Quality improvement strategies that have targeted decreasing the number of PICC lumens have reduced complications and healthcare costs. ${ }^{17-19}$ However, variability exists in the selection of the number of PICC lumens, and many providers request multilumen devices "just in case" additional lumens are needed. ${ }^{20,21}$ Such variation in device selection may stem from the paucity of information that defines the appropriate indications for the use of singleversus multilumen PICCs.

*Corresponding Author: Vineet Chopra MD, MSc; E-mail: vineetc@umich.edu

Received: April 27, 2018; Revised: September 2, 2018;

Accepted: September 12, 2018

@ 2019 Society of Hospital Medicine DOI 10.12788/jhm.3097
Therefore, to ensure appropriateness of PICC use, we designed an intervention to improve selection of the number of PICC lumens.

\section{METHODS}

We conducted this pre-post quasi-experimental study in accordance with SQUIRE guidelines. ${ }^{22}$ Details regarding clinical parameters associated with the decision to place a PICC, patient characteristics, comorbidities, complications, and laboratory values were collected from the medical records of patients. All PICCs were placed by the Vascular Access Service Team (VAST) during the study period.

\section{Intervention}

The intervention consisted of three components: first, all hospitalists, pharmacists, and VAST nurses received education in the form of a CME lecture that emphasized use of the Michigan Appropriateness Guide for Intravenous Catheters (MAGIC). ${ }^{1}$ These criteria define when use of a PICC is appropriate and emphasize how best to select the most appropriate device characteristics such as lumens and catheter gauge. Next, a multidisciplinary task force that consisted of hospitalists, VAST nurses, and pharmacists developed a list of indications specifying when use of a multilumen PICC was appropriate. ${ }^{1}$ Third, the order for a PICC in our electronic medical record (EMR) system was modified to set single-lumen PICCs as default. If a multilumen PICC was requested, text-based justification from the ordering clinician was required.

As an additional safeguard, a VAST nurse reviewed the num- 
ber of lumens and clinical scenario for each PICC order prior to insertion. If the number of lumens ordered was considered inappropriate on the basis of the developed list of MAGIC recommendations, the case was referred to a pharmacist for additional review. The pharmacist then reviewed active and anticipated medications, explored options for adjusting the medication delivery plan, and discussed these options with the ordering clinician to determine the most appropriate number of lumens.

\section{Measures and Definitions}

In accordance with the criteria set by the Centers for Disease Control National Healthcare Safety Network, ${ }^{23}$ CLABSI was defined as a confirmed positive blood culture with a PICC in place for 48 hours or longer without another identified infection source or a positive PICC tip culture in the setting of clinically suspected infection. Venous thrombosis was defined as symptomatic upper extremity deep vein thromboembolism or pulmonary embolism that was radiographically confirmed after the placement of a PICC or within one week of device removal. Catheter occlusion was captured when documented or when TPA was administered for problems related to the PICC. The appropriateness of the number of PICC lumens was independently adjudicated by an attending physician and clinical pharmacist by comparing the indications of the device placed against predefined appropriateness criteria.

\section{Outcomes}

The primary outcome of interest was the change in the proportion of single-lumen PICCs placed. Secondary outcomes included (1) the placement of PICCs with an appropriate number of lumens, (2) the occurrence of PICC-related complications (CLABSI, venous thrombosis, and catheter occlusion), and (3) the need for a second procedure to place a multilumen device or additional vascular access.

\section{Statistical Analysis}

Descriptive statistics were used to tabulate and summarize patient and PICC characteristics. Differences between pre- and postintervention populations were assessed using $\chi^{2}$, Fishers exact, $t$, and Wilcoxon rank sum tests. Differences in complications were assessed using the two-sample tests of proportions. Results were reported as medians (IQR) and percentages with corresponding 95\% confidence intervals. All statistical tests were two-sided, with $P<.05$ considered statistically significant. Analyses were conducted with Stata v.14 (stataCorp, College Station, Texas).

\section{Ethical and Regulatory Oversight}

This study was approved by the Institutional Review Board at the University of Michigan (IRB\#HUM00118168).

\section{RESULTS}

Of the 133 PICCs placed preintervention, 64.7\% $(n=86)$ were single lumen, $33.1 \%(n=44)$ were double lumen, and $2.3 \%$ ( = 3) were triple lumen. Compared with the preintervention period, the use of single-lumen PICCs significantly increased following the intervention (64.7\% to $93.6 \%$; $P<.001$; Figure 1).

\section{Simultaneous administration of multiple incompatible medications}

TPN infusion with concurrent need for additional IV medications

Simultaneous use of continuous vesicant or irritant chemotherapy with other medications

Double Lumen: IV tacrolimus, IV fosarnet, IV cytarabine or doxorubicin/vincristine as a combined infusion with simultaneous use of additional IV medications.

Triple Lumen: continuous vesicant or irritant chemotherapy meeting the requirement for a double lumen PICC plus actively receiving blood products.

\section{Need for vasopressors}

ie continuous use of phenylephrine, vasopressin, dopamine, norepinephrine, epinephrine, dobutamine, milrinone.

FIG 1. Michigan Multilumen PICC Criteria

Abbreviations: IV, intravenous; PICC, peripherally inserted central catheter; TPN, total parenteral nutrition.

As well, the proportion of PICCs with an inappropriate number of lumens decreased from $25.6 \%$ to $2.2 \%(P<.001$; Table 1$)$.

Preintervention, $14.3 \%(95 \% \mathrm{Cl}=8.34-20.23)$ of the patients with PICCs experienced at least one complication $(n=19)$. Following the intervention, $15.1 \%(95 \% \mathrm{Cl}=7.79-22.32)$ of the 93 patients with PICCs experienced at least one complication (absolute difference $=0.8 \%, P=.872$ ). With respect to individual complications, CLABSI decreased from 5.3\% ( $n=7 ; 95 \%$ $\mathrm{Cl}=1.47-9.06)$ to $2.2 \%(\mathrm{n}=2 ; 95 \% \mathrm{Cl}=-0.80-5.10 ; P=.239)$. Similarly, the incidence of catheter occlusion decreased from $8.3 \%(\mathrm{n}=11 ; 95 \% \mathrm{Cl}=3.59-12.95)$ to $6.5 \%(\mathrm{n}=6 ; 95 \% \mathrm{Cl}=$ $1.46-11.44 ; P=.610 ;$ Table). Notably, only $12.1 \%(n=21)$ of patients with a single-lumen PICC experienced any complication, whereas $20.0 \%(n=10)$ of patients with a double lumen, and $66.7 \%(n=2)$ with a triple lumen experienced a PICC-associated complication $(P=.022)$. Patients with triple lumens had a significantly higher incidence of catheter occlusion compared with patients that received double- and single-lumen PICCs (66.7\% vs. $12.0 \%$ and $5.2 \%$, respectively; $P=.003$ ).

No patient who received a single-lumen device required a second procedure for the placement of a device with additional lumens. Similarly, no documentation suggesting an insufficient number of PICC lumens or the need for additional vascular access (eg, placement of additional PICCs) was found in medical records of patients postintervention. Pharmacists supporting the interventions and VAST team members reported no disagreements when discussing number of lumens or appropriateness of catheter choice.

\section{DISCUSSION}

In this single center, pre-post quasi-experimental study, a multimodal intervention based on the MAGIC criteria significantly reduced the use of multilumen PICCs. Additionally, a trend toward reductions in complications, including CLABSI and catheter occlusion, was also observed. Notably, these changes in ordering practices did not lead to requests for additional devices or replacement with a multilumen PICC when a single-lumen device was inserted. Collectively, our findings suggest that the use of single-lumen devices in a large direct care service can be feasibly 
TABLE. Patient Characteristics and Complications

\begin{tabular}{|c|c|c|c|}
\hline Patient Characteristic, n (\%) & $\begin{array}{l}\text { Preintervention } \\
\quad(n=133)\end{array}$ & $\begin{array}{l}\text { Postintervention } \\
\quad(n=93)\end{array}$ & $P$ Value \\
\hline Age, mean (SD) & $60.9(1.5)$ & $60.3(1.8)$ & .802 \\
\hline Female & $72(54.1)$ & $38(40.9)$ & .049 \\
\hline Charlson, median (IQR) & $4(2-6)$ & $4(2-7)$ & .678 \\
\hline Smoking status & & & .321 \\
\hline Never & $56(43.4)$ & $37(40.2)$ & \\
\hline Former & $54(41.9)$ & $42(45.7)$ & \\
\hline Current & $19(14.7)$ & $13(14.1)$ & \\
\hline Body mass index, mean (SD) & $30.7(0.9)$ & $29.6(1.2)$ & .455 \\
\hline Length of stay, median (IQR) & $8(5-13)$ & $7(5-13)$ & .429 \\
\hline Ever ICU stay & $5(3.8)$ & $4(4.3)$ & 1.00 \\
\hline History of CLABSI and/or VTE & $27(20.3)$ & $16(17.2)$ & .559 \\
\hline \multicolumn{4}{|l|}{ Lab values, mean (SD) } \\
\hline White blood cell count & $9.4(1.0)$ & $10.1(0.7)$ & .575 \\
\hline Absolute neutrophils & $7.3(0.8)$ & $7.7(0.5)$ & .732 \\
\hline Estimated Glomerular Filtration Rate (eGFR) & $56.4(0.9)$ & $59.0(0.4)$ & .018 \\
\hline $\mathrm{eGFR}<45$ & $118(88.7)$ & $92(98.92)$ & .003 \\
\hline \multicolumn{4}{|l|}{ Medications } \\
\hline Systemic anticoagulant & $132(99.3)$ & $93(100)$ & .402 \\
\hline Antiplatelet medication & $49(36.8)$ & $35(37.6)$ & .903 \\
\hline \multicolumn{4}{|l|}{ PICC Characteristics, n (\%) } \\
\hline Lumens & & & $<.001$ \\
\hline Single & $86(64.7)$ & $87(93.6)$ & \\
\hline Double & $44(33.1)$ & $6(6.5)$ & \\
\hline Triple & $3(2.3)$ & 0 & \\
\hline Inappropriate PICC selection & $34(25.6)$ & $2(2.2)$ & $<.001$ \\
\hline \multicolumn{4}{|l|}{ Complications, n (\%) (95\% Cl) } \\
\hline CLABSI & $7(5.26)(1.47-9.06)$ & $2(2.15)(-0.80-5.10)$ & .239 \\
\hline VTE & $4(3.01)(0.10-5.91)$ & $7(7.53)(2.16-12.89)$ & .120 \\
\hline Catheter occlusion & $11(8.27)(3.59-12.95)$ & $6(6.45)(1.46-11.44)$ & .610 \\
\hline Any complication & 19 (14.29) (8.34-20.23) & $14(15.1)(7.79-22.32)$ & .872 \\
\hline
\end{tabular}

Abbreviations: CLABSI, central line-associated bloodstream infection; ICU, intensive care unit; IQR, interquartile range; PICC, peripherally inserted central catheter; SD, standard deviation; VTE, venous thromboembolism

and safely increased through this approach. Larger scale studies that implement MAGIC to inform placement of multilumen PICCs and reduce PICC-related complications now appear necessary.

The presence of a PICC, even for short periods, significantly increases the risk of CLABSI and is one of the strongest predictors of venous thrombosis risk in the hospital setting. ${ }^{19,24,25}$ Although some factors that lead to this increased risk are patient-related and not modifiable (eg, malignancy or intensive care unit status), increased risk linked to the gauge of PICCs and the number of PICC lumens can be modified by improving device selection. ${ }^{9,18,26}$ Deliberate use of PICCs with the least numbers of clinically necessary lumens decreases risk of CLABSI, venous thrombosis, and overall cost. ${ }^{17,19,26}$ Additionally, greater rates of occlusion with each additional PICC lumen may result in the interruption of intravenous therapy, the administration of costly medications (eg, tissue plasminogen ac- tivator) to salvage the PICC, and premature removal of devices should the occlusion prove irreversible. ${ }^{8}$

We observed a trend toward decreased PICC complications following implementation of our criteria, especially for the outcomes of CLABSI and catheter occlusion. Given the pilot nature of this study, we were underpowered to detect a statistically significant change in PICC adverse events. However, we did observe a statistically significant increase in the rate of single-lumen PICC use following our intervention. Notably, this increase occurred in the setting of high rates of single-lumen PICC use at baseline (64\%). Therefore, an important takeaway from our findings is that room for improving PICC appropriateness exists even among high performers. In turn, high baseline use of single-lumen PICCs may also explain why a robust reduction in PICC complications was not observed in our study, given that other studies showing reduction in the rates of complications began with considerably 


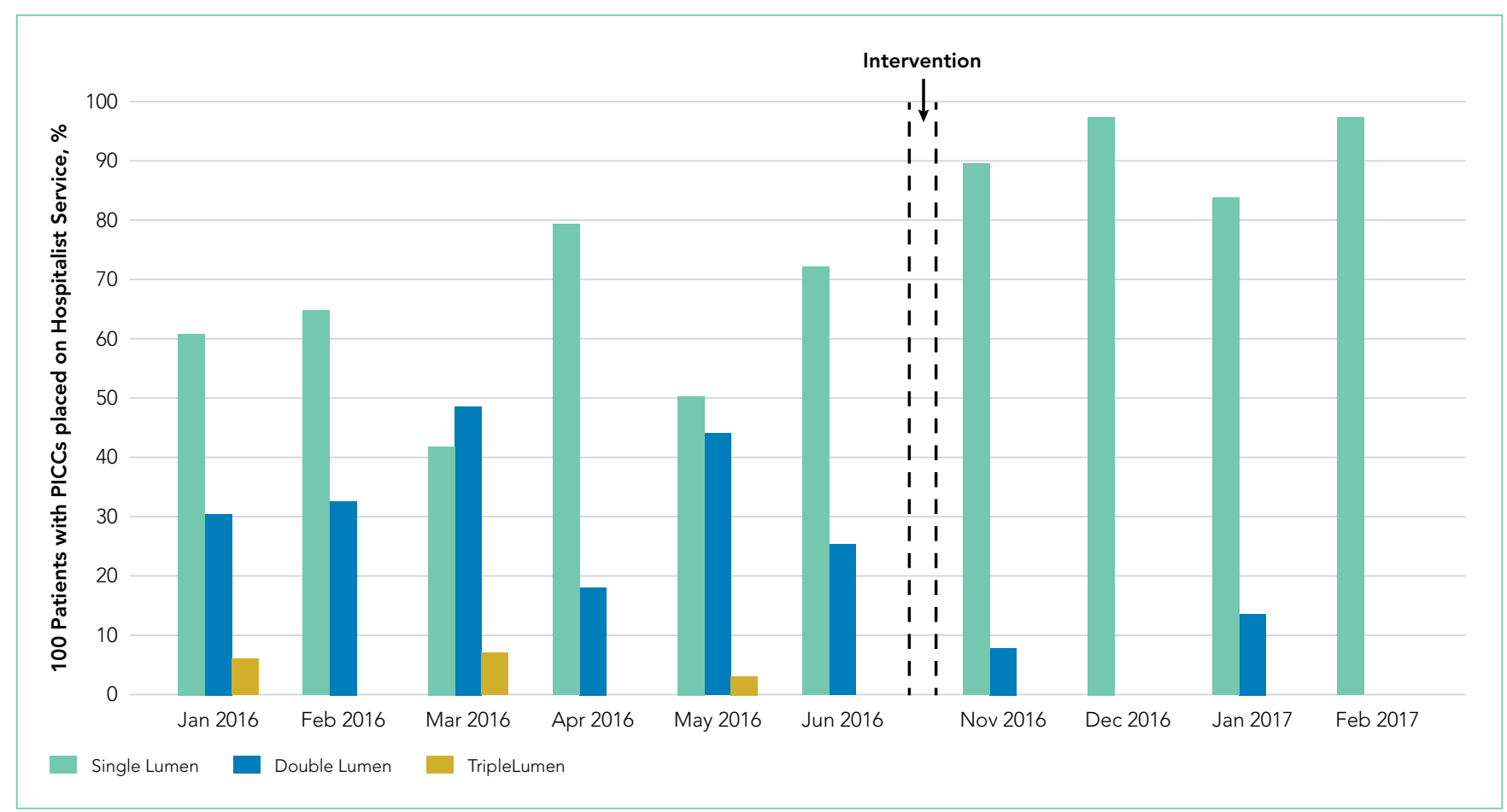

FIG 2. Utilization of PICCs by Number of Lumens, from Pre- to Postintervention. Abbreviation: PICC, peripherally inserted central catheter

low rates of single-lumen device use. ${ }^{19}$ Outcomes may improve, however, if we expand and sustain these changes or expand to larger settings. For example, (based on assumptions from a previously published simulation study and our average hospital medicine daily census of 98 patients) the increased use of single- over multilumen PICCs is expected to decrease CLABSI events and venous thrombosis episodes by 2.4 -fold in our hospital medicine service with an associated cost savings of $\$ 74,300$ each year. ${ }^{17}$ Additionally, we would also expect the increase in the proportion of single-lumen PICCs to reduce rates of catheter occlusion. This reduction, in turn, would lessen interruptions in intravenous therapy, the need for medications to treat occlusion, and the need for device replacement all leading to reduced costs. ${ }^{27}$ Overall, our intervention (informed by appropriateness criteria) provides substantial benefits to hospital savings and patient safety.

After our intervention, 98\% of all PICCs placed were found to comply with appropriate criteria for multilumen PICC use. We unexpectedly found that the most important factor driving our findings was not oversight or order modification by the pharmacy team or VAST nurses, but rather better decisions made by physicians at the outset. Specifically, we did not find a single instance wherein the original PICC order was changed to a device with a different number of lumens after review from the VAST team. We attribute this finding to receptiveness of physicians to change ordering practices following education and the redesign of the default EMR PICC order, both of which provided a scientific rationale for multilumen PICC use. Clarifying the risk and criteria of the use of multilumen devices along with providing an EMR ordering process that supports best practice helped hospitalists "do the right thing." Additionally, setting single-lumen devices as the preselected EMR order and requiring text-based justification for placement of a multilumen PICC helped provide a nudge to physicians, much as it has done with antibiotic choices. ${ }^{28}$

Our study has limitations. First, we were only able to identify complications that were captured by our EMR. Given that over $70 \%$ of the patients in our study were discharged with a PICC in place, we do not know whether complications may have developed outside the hospital. Second, our intervention was resource intensive and required partnership with pharmacy, VAST, and hospitalists. Thus, the generalizability of our intervention to other institutions without similar support is unclear. Third, despite an increase in the use of single-lumen PICCs and a decrease in multilumen devices, we did not observe a significant reduction in all types of complications. While our high rate of single-lumen PICC use may account for these findings, larger scale studies are needed to better study the impact of MAGIC and appropriateness criteria on PICC complications. Finally, given our approach, we cannot identify the most effective modality within our bundled intervention. Stepped wedge or single-component studies are needed to further address this question.

In conclusion, we piloted a multimodal intervention to promote the use of single-lumen PICCs while lowering the use of multilumen devices. By using MAGIC to create appropriate indications, the use of multilumen PICCs declined and complications trended downwards. Larger, multicenter studies to validate our findings and examine the sustainability of this intervention would be welcomed.

Disclosures: The authors have nothing to disclose. 


\section{References}

1. Chopra V, Flanders SA, Saint S, et al. The Michigan Appropriateness Guide for Intravenous Catheters (MAGIC): Results from a multispecialty panel using the RAND/UCLA appropriateness method. Ann Intern Med. 2015;163(6 Suppl):S1-S40. doi: 10.7326/M15-0744.

2. Taylor RW, Palagiri AV. Central venous catheterization. Crit Care Med. 2007;35(5):1390-1396. doi: 10.1097/01.CCM.0000260241.80346.1B.

3. Pikwer A, Akeson J, Lindgren S. Complications associated with peripheral or central routes for central venous cannulation. Anaesthesia. 2012;67(1):65-71. doi: 10.1111/j.1365-2044.2011.06911.x.

4. Johansson $\mathrm{E}$, Hammarskjold F, Lundberg D, Arnlind MH. Advantages and disadvantages of peripherally inserted central venous catheters (PICC) compared to other central venous lines: a systematic review of the literature. Acta Onco. 2013;52(5):886-892. doi: 10.3109/0284186X.2013.773072.

5. Pan L, Zhao $Q$, Yang $X$. Risk factors for venous thrombosis associated with peripherally inserted central venous catheters. Int J Clin Exp Med. 2014;7(12):5814-5819.

6. Herc E, Patel P, Washer LL, Conlon A, Flanders SA, Chopra V. A model to predict central-line-associated bloodstream infection among patients with peripherally inserted central catheters: The MPC score. Infect Cont Hosp Ep. 2017;38(10):1155-1166. doi: 10.1017/ice.2017.167

7. Maki DG, Kluger DM, Crnich CJ. The risk of bloodstream infection in adults with different intravascular devices: a systematic review of 200 published prospective studies. Mayo Clin Proc. 2006;81(9):1159-1171. doi: 10.4065/81.9.1159

8. Smith SN, Moureau N, Vaughn VM, et al. Patterns and predictors of peripherally inserted central catheter occlusion: The 3P-O study. J Vasc Interv Radiol. 2017;28(5):749-756.e742. doi: 10.1016/j.jvir.2017.02.005.

9. Chopra V, Anand S, Hickner A, et al. Risk of venous thromboembolism associated with peripherally inserted central catheters: a systematic review and meta-analysis. Lancet. 2013;382(9889):311-325. doi: 10.1016/S0140 6736(13)60592-9.

10. Chopra V, Ratz D, Kuhn L, Lopus T, Lee A, Krein S. Peripherally inserted central catheter-related deep vein thrombosis: contemporary patterns and predictors. J Thromb Haemost. 2014;12(6):847-854. doi: 10.1111/jth.12549.

11. Carter JH, Langley JM, Kuhle S, Kirkland S. Risk factors for central venous catheter-associated bloodstream infection in pediatric patients: A cohort study. Infect Control Hosp Epidemiol. 2016;37(8):939-945. doi: 10.1017/ ice.2016.83.

12. Chopra V, Ratz D, Kuhn L, Lopus T, Chenoweth C, Krein S. PICC-associated bloodstream infections: prevalence, patterns, and predictors. Am J Med. 2014;127(4):319-328. doi: 10.1016/j.amjmed.2014.01.001.

13. O'Grady NP, Alexander M, Burns LA, et al. Guidelines for the prevention of intravascular catheter-related infections. Clin Infect Dis. 2011;52(9):e162-e193. doi: 10.1093/cid/cir257

14. Parkinson R, Gandhi M, Harper J, Archibald C. Establishing an ultrasound guided peripherally inserted central catheter (PICC) insertion service. Clin Radiol. 1998;53(1):33-36. doi: 10.1016/S0009-9260(98)80031-7.
15. Shannon RP, Patel B, Cummins D, Shannon AH, Ganguli G, Lu Y. Economics of central line--associated bloodstream infections. Am J Med Qual. 2006;21(6 Suppl):7s-16s. doi: 10.1177/1062860606294631

16. Mermis JD, Strom JC, Greenwood JP, et al. Quality improvement initiative to reduce deep vein thrombosis associated with peripherally inserted central catheters in adults with cystic fibrosis. Ann Am Thorac Soc. 2014;11(9):14041410. doi: 10.1513/AnnalsATS.201404-175OC.

17. Ratz D, Hofer T, Flanders SA, Saint S, Chopra V. Limiting the number of lumens in peripherally inserted central catheters to improve outcomes and reduce cost: A simulation study. Infect Control Hosp Epidemiol. 2016;37(7):811817. doi: 10.1017/ice.2016.55.

18. Chopra V, Anand S, Krein SL, Chenoweth C, Saint S. Bloodstream infection, venous thrombosis, and peripherally inserted central catheters: reappraising the evidence. Am J Med. 2012;125(8):733-741. doi: 10.1016/j.amjmed.2012.04.010

19. O'Brien J, Paquet F, Lindsay R, Valenti D. Insertion of PICCs with minimum number of lumens reduces complications and costs. J Am Coll Radiol. 2013;10(11):864-868. doi: 10.1016/j.jacr.2013.06.003

20. Tiwari MM, Hermsen ED, Charlton ME, Anderson JR, Rupp ME. Inappropriate intravascular device use: a prospective study. J Hosp Infect. 2011;78(2):128132. doi: 10.1016/j.jhin.2011.03.004.

21. Chopra V, Kuhn L, Flanders SA, Saint S, Krein SL. Hospitalist experiences, practice, opinions, and knowledge regarding peripherally inserted central catheters: results of a national survey. J Hosp Med. 2013;8(11):635-638. doi: 10.1002/jhm.2095

22. Goodman D, Ogrinc G, Davies L, et al. Explanation and elaboration of the SQUIRE (Standards for Quality Improvement Reporting Excellence) Guidelines, V.2.0: examples of SQUIRE elements in the healthcare improvement literature. BMJ Qual Saf. 2016;25(12):e7. doi: 10.1136/bmjqs-2015-004480.

23. CDC Bloodstream Infection/Device Associated Infection Module. https://wwwcdcgov/nhsn/pdfs/pscmanual/4psc_clabscurrentpdf 2017. Accessed April 11, 2017.

24. Woller SC, Stevens SM, Jones JP, et al. Derivation and validation of a simple model to identify venous thromboembolism risk in medical patients. Am J Med. 2011;124(10):947-954.e2. doi: 10.1016/j.amjmed.2011.06.004.

25. Paje D, Conlon A, Kaatz S, et al. Patterns and predictors of short-term peripherally inserted central catheter use: A multicenter prospective cohort study. $J$ Hosp Med. 2018;13(2):76-82. doi: 10.12788/jhm.2847.

26. Evans RS, Sharp JH, Linford LH, et al. Reduction of peripherally inserted central catheter-associated DVT. Chest. 2013;143(3):627-633. doi: 10.1378/ chest.12-0923

27. Smith S, Moureau N, Vaughn VM, et al. Patterns and predictors of peripherally inserted central catheter occlusion: The 3P-O study. J Vasc Interv Radiol. 2017:28(5):749-756.e2. doi: 10.1016/j.jvir.2017.02.005

28. Vaughn VM, Linder JA. Thoughtless design of the electronic health record drives overuse, but purposeful design can nudge improved patient care. BMJ Qual Saf. 2018;27(8):583-586. doi: 10.1136/bmjqs-2017-007578. 